\title{
Heparinized chitosan/hydroxyapatite scaffolds stimulate angiogenesis
}

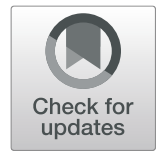

\author{
Griselda V. Nájera-Romero ${ }^{1}$, Muhammad Yar $^{2}$ and Ihtesham Ur Rehman ${ }^{1 *}$ (D)
}

\begin{abstract}
Formation of blood vessels during bone regeneration represents a major challenge for tissue engineered constructs. Poor revascularization can lead to scaffold failure and consequently, leads to non-healing fracture. Heparin is known to bind with angiogenic growth factors influencing the process of new blood vessels formation. There are several problems associated with the use of growth factors in clinic such as low stability, uncontrolled delivery to the site, and high price. The aim of the present study was to explore the potential of heparin to produce pro-angiogenic bone regeneration materials. Chitosan/hydroxyapatite freeze-gelled scaffolds were prepared and loaded with heparin. Different concentrations of heparin were successfully loaded onto the scaffolds, its release from the scaffold was analysed by toluidine blue assay and their angiogenic effect was evaluated by chorioallantoic membrane (CAM) assay to determine the optimal concentration of heparin to induce a proangiogenic effect. It was noted that low heparin concentrations exhibited a positive effect, with approximately $28 \mathrm{\mu g}$ per scaffold indicating a significant increment in blood vessels. The synthesized materials showed no cytotoxic effects when evaluated by using U2OS cell line.
\end{abstract}

Keywords: Angiogenesis, Bone regeneration, Heparin, Chitosan, Bioactive

\section{Introduction}

Bone possesses the capacity to repair itself whenever an injury occurs. Successful bone healing depends on several factors that include, age, nutrition and, type of fracture among others $[1,2]$. However, in some cases of tumour removal, traumatic fractures, or injury infection, complications may arise, resulting in critical size bone defects where the loss of bone exceeds its self-healing ability. One of the challenges of current bone treatment is the stimulation of blood vessel development $[3,4]$. Revascularization during bone regeneration is key to a proper outcome, as blood vessels provide nourishment, excretion pathways, and allow progenitor cells to reach from their niches to the injured site. Angiogenesis is the generation of blood vessels from pre-existing vasculature. Therefore, for bone tissue engineering, a wide range of options have been investigated to approach the limitation of enhancing angiogenesis during bone repair $[4,5]$.

\footnotetext{
* Correspondence: i.u.rehman@lancaster.ac.uk

'Engineering Department, Faculty of Science and Technology, Lancaster University, Lancaster LA1 4YW, UK

Full list of author information is available at the end of the article
}

Currently, delivery of growth factors has been favoured, as they highly influence the course of both processes: angiogenesis and osteogenesis. Vascular endothelial growth factor (VEGF) and fibroblast growth factor (FGF) families have been preferred for these purposes. However, the direct use of these biomolecules has presented some drawbacks such as: high costs, unknown doses, contradictory results or possible tumour inducement, which have raised doubts concerning their applicability as a completely effective therapy for neovascularization during bone regeneration [4-7]. Researchers are continuously looking to improve effectiveness of growth factors and the search for alternatives remains a topic of enormous interest.

Heparin has proven to be very effective on binding with VEGF and other growth factors [8-14]. Heparin is a sulphated glycosaminoglycan better known for its anticoagulant properties, but it has also the ability to interact with different proteins due to its anionic nature [15, 16]. Chiodelli et al. (2015) published an extensive review on heparin and heparan sulphate proteoglycans molecular interactions related to revascularization. In this 
review, the authors highlight heparin ability to bind with angiogenic growth factors (AGFs), which include both inhibitors and promoters. The balance between these two is the way heparin aids neovascularization regulation. In addition, it has been stated that the group $6 \mathrm{SO}_{3}$ of heparin is responsible for the interaction with VEGF [17].

Furthermore, Bhakuni et al. (2016) and Rema et al. (2003) reported that molecular weight of heparin plays an important role on its angiogenic activity $[18,19]$. According to Rema et al. (2003) and Collen et al. (2000), low molecular weight heparin (LMWH) has shown to inhibit angiogenesis compared to high molecular weight heparin $(\mathrm{HMWH})$ on a chorioallantoic membrane assay (CAM assay) [19, 20]. Ito \& Welsh's (1999) reported on dual effects of heparin in the affinity of VEGF for its receptors, observing a difference in the action according to length of the polysaccharide unit [21]. Nonetheless, this behaviour seems to be completely dependent on the angiogenic regulator's affinity and molecular requirements.

Regardless of the vast information available regarding the use of heparin to bind growth factors to scaffolds, not many studies regarding the angiogenic influence of heparin itself on the bone healing process have been reported. Additionally, the combination of chitosan/hydroxyapatite/heparin without external growth factors has not been investigated for bone angiogenesis promotion. Recently, our research group reported on the use of heparin loaded hydrogels as an angiogenic promoter for wound healing treatments [2224]. On the other hand, Gümüșderelioğlu \& Aday (2011) reported the osteogenic activity of heparinised chitosan scaffolds promoting in vitro proliferation and differentiation of pre-osteoblasts cells [25]. Therefore, the purpose of this study is to evaluate the angiogenic effect of heparin concentration present on bioactive scaffolds fabricated by employing chitosan and hydroxyapatite.

Chitosan is a polysaccharide that has been very much exploited as a biomaterial due to its remarkable behaviour within the body, which includes excellent biocompatibility, tuneable biodegradability, no toxicity and good cell adhesion (optimal for cell proliferation) [26-28]. Likewise, hydroxyapatite is a bioceramic that has been used in bone repair and regeneration due to its excellent biocompatibility, bioresorption and bioactivity [29, 30]. Reported scaffolds composed of combinations of these materials have demonstrated potential characteristics to be used in bone tissue engineering since they provide a porous matrix, which enables vascular infiltration and as well as bone tissue growth $[26,31]$.

In this study, porous scaffolds from chitosan and hydroxyapatite were prepared using freeze-gelation method and loaded with variable concentrations of heparin. Our hypothesis is that the heparin present in the scaffold will interact with the angiogenic growth factors from the surroundings and will induce a pro-angiogenic response.

\section{Materials and methodology Materials}

Materials used in this study were chitosan, molecular weight $100,000-300,000$, DD $\geq 90 \%$. $\left(\right.$ ACROS organics $^{\mathrm{m}}$ ). Hydroxyapatite sintered powder (Captal $\left.S^{\circ}\right)$, particle size of the used hydroxyapatite varied from $0.5 \pm 2$ to $12 \pm 1 \mu \mathrm{m}$ [32] . Acetic acid glacial $\geq 99.85 \%$ (Sigma Aldrich). Sodium hydroxide $(\mathrm{NaOH}) 98 \%$ pellets (ACROS Organics $\left.{ }^{\mathrm{Tm}}\right)$. Phosphate buffered saline tablets $\left(\mathrm{Oxoid}^{\mathrm{m}}\right)$ ). Absolute ethanol (AnalR NORMAPUR). Heparin sodium salt, from porcine intestinal mucosa, IU $>=100 / \mathrm{mg}$ (Alfa Aesar). Fertilized chicken eggs (Henry Stewart Co. Ltd., UK). Toluidine blue O dye (Sigma Aldrich). Hydrochloric acid 1N (Sigma Aldrich) and sodium chloride (ACROS Organics ${ }^{\mathrm{nt}}$ ).

\section{Preparation of scaffolds}

All scaffolds were prepared according to the procedure reported earlier by our research group Qasim S. B. et al. (2015) [32], with slight modifications to incorporate heparin sodium salt.

Chitosan ( $7.5 \mathrm{~g}$ ) was added to a $250 \mathrm{~mL}$ volumetric flask containing warmed distilled water. This mixture was stirred for $1 \mathrm{~h}$, followed by the addition of glacial acetic acid dropwise at an approximate rate of $1 \mathrm{~mL} / 5$ min, until a solution $0.3 \mathrm{M}$ was achieved. Obtained chitosan solution was a golden colour viscous liquid.

The chitosan solution was diluted to $250 \mathrm{~mL}$ with distilled water while maintaining stirring for another $6 \mathrm{~h}$. To this solution hydroxyapatite powder $(7.5 \mathrm{~g})$ was added and the mixture was further stirred over night for approximately another $13 \mathrm{~h}$.

The solution was transferred into plastic petri dishes, adding the necessary amount according to the required scaffold thickness. The containers were placed into the freezer at $4{ }^{\circ} \mathrm{C}$ for $1.5 \mathrm{~h}$. After this period, the temperature was gradually decreased at a rate of $-4{ }^{\circ} \mathrm{C}$ per hour until a required temperature of $-20^{\circ} \mathrm{C}$ was reached. The frozen solution was kept at $-20^{\circ} \mathrm{C}$ for further $12 \mathrm{~h}$, which generated frozen discs.

Subsequently, the frozen discs were carefully removed from the petri dishes by pressing the edges of the mould and by carefully applying pressure at the bottom of the dish. A $3 \mathrm{M} \mathrm{NaOH} /$ ethanol solution was poured into glass petri dishes and the frozen discs were placed into them, more $\mathrm{NaOH}$ solution was poured into the glass petri dish to cover completely the frozen disc. All the immersed discs were placed at $-20^{\circ} \mathrm{C}$ for further $12 \mathrm{~h}$.

After $12 \mathrm{~h}$ of immersion in the gelling solution the discs were taken out and washed as follows: 15 min with distilled water, 5 min with PBS, 15 min in $80 \%$ ethanol solution and $15 \mathrm{~min}$ in absolute ethanol.

Finally, the obtained product was left open to dry at room temperature in a well-ventilated fume cupboard overnight (approximately $13 \mathrm{~h}$ ). 


\section{Heparin loading}

Scaffolds were cut into disc shapes of approximately 5 $\mathrm{mm}$ diameter using a hole puncher or a cork borer. Solutions of heparin with concentrations of 0.5, 1, 2 and 5 $\mathrm{mg} / \mathrm{mL}$ were prepared by dissolving heparin sodium salt in the correspondent amount of deionized water. The scaffold discs were immersed for $1.5 \mathrm{~h}$ in $50 \mathrm{~mL}$ of the heparin solution followed by freeze-drying at $-25{ }^{\circ} \mathrm{C}$ for $24 \mathrm{~h}$ to remove the moisture.

\section{Analysing morphology of scaffolds}

To access the porous size and connectivity of fabricated scaffolds, scanning electron microscopy (SEM), Jeol JSM $7800 \mathrm{~F}$ microscope was used to study morphologies. Samples were cut into half in such a way that not only the bases of the disc but also the edges could also be analysed. Each sample holder contained 3 pieces of each concentration: one placed with the upper base (chitosan side) facing up, another with the bottom base (hydroxyapatite side) facing up and the third one with the edge of the scaffold facing up. The samples were gold sputtered with a thickness of 10 $\mathrm{nm}$. Porosity of the samples was determined by measuring

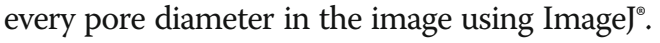

\section{Chemical structural analysis using Fourier-transform infrared spectroscopy (FTIR)}

FTIR characterization was performed using a Thermo Nicolet iS50 FTIR spectrometer in conjunction with Attenuated Total Reflectance (ATR) sampling accessory having a diamond crystal, which allows to analyse surface of the sample. Spectra from the upper and bottom of the disc scaffolds were taken for comparison. Spectra were obtained at $4 \mathrm{~cm}^{-1}$ resolution by accumulating 128 number of scans using Omnic $9^{\mathrm{TM}}$ software.

\section{Heparin loading determination}

Toluidine Blue assay was developed by Smith P. K. et al. (1980) [33]. The assay is based on the colour change from blue to purple, which occurs when toluidine blue interacts with heparin containing solution. The purple shade and the amount of heparin-dye complex produced depend on the concentration of heparin, thus the presence of heparin is not only visibly determined, but it also can be quantified by comparing with a calibration curve.

Two experiments were performed. The first one was the visual determination of heparin in the scaffold, i. e. the scaffolds were immersed in toluidine blue solution to observe the colour change. The second experiment was to quantify the maximum amount of heparin contained in the scaffold.

For both evaluations, a $0.05 \%$ toluidine blue solution was used. This solution was prepared in $100 \mathrm{~mL}$ volumetric flask by dissolving $5 \mathrm{mg}$ of toluidine blue $\mathrm{O}$ dye in an aqueous solution containing $0.2 \mathrm{~g}$ of $\mathrm{NaCl}$ and $1 \mathrm{~mL}$ of $1 \mathrm{~N} \mathrm{HCl}$.

\section{Qualitative determination}

A set of 2 scaffolds from each heparin concentration was immersed separately in $1.5 \mathrm{~mL}$ of the $0.05 \%$ toluidine blue solution. The scaffolds were left to rest for $5 \mathrm{~min}$, followed by washing with deionized water several times to ensure that the dye was fully removed. The scaffolds were carefully dried using a tissue paper. Pictures of each scaffold were taken to verify the presence and distribution of the heparin on the scaffold by observing the purple colouration.

\section{Heparin content estimation}

For heparin evaluation, eight time-intervals were selected; 30, 60, 90, 150 and $210 \mathrm{~min}, 1,2$ and 5 days. The study consisted of 3 sets of experiments with 2 replicates per each sample concentration The same set of samples (which includes all the heparin concentrations) was evaluated on each designated interval. The cylindrical scaffolds were individually placed into test tubes adding 1 $\mathrm{mL}$ of deionized water into each tube, followed by incubation at $37^{\circ} \mathrm{C}$ for set time intervals as mentioned above.

The samples were taken out from the incubator at set time intervals and $250 \mu \mathrm{L}$ of the incubation water were extracted from every sample tube ${ }^{* * *}$ and added into correspondent tubes containing $750 \mu \mathrm{L}$ of toluidine blue solution. Every tube was carefully swirled, so the incubation water is completely mixed with the toluidine blue solution. This process was followed by resting the solution for $30 \mathrm{~min}$ at room temperature, adding $750 \mu \mathrm{L}$ of n-hexane into every tube and vigorously shaking the mixture manually for $30 \mathrm{~s}$. Once the mixture has settled, the aqueous phase (bottom liquid) was removed and transferred into a clean 96 well plate for its reading. The absorbance of this phase was measured at $630 \mathrm{~nm}$ using a plate reader, the readings were compared to the results of a calibration curve previously prepared.

**NOTE: Once the $250 \mu \mathrm{L}$ of incubation water was extracted, the samples were changed to new testing tubes with clean deionized water and taken back to the incubator for set times of the experiment until the completion of the process.

\section{In-vitro degradation}

The procedure employed for the biodegradation in this study is based on the method reported earlier by our research group Qasim S. (2017) [34].

Four time-intervals were evaluated: 1, 7, 14 and 21 days. Independent sets of samples were prepared for each time-interval.

The media solution used for this experiment was prepared by dissolving $500 \mathrm{mg}$ of lysozyme into $500 \mathrm{~mL}$ of a sterile PBS solution. The scaffolds were individually weighed, recording this value as Wi. Subsequently, each 
sample was placed into individual test tubes containing $3 \mathrm{~mL}$ of the lysozyme solution and incubated at $37^{\circ} \mathrm{C}$. Lysozyme solution was replaced every week. Once the correspondent time interval has been concluded, the set of samples was taken out of the incubator. The $\mathrm{pH}$ of the media was measured and recorded. The samples were taken out from the media and carefully washed with deionized water. Drying of the sample was achieved by sandwiching the sample between two water absorbing sheets of tissue paper for $30 \mathrm{~s}$ to remove moisture from the surface. Dried samples were transferred into clean individual vials for $24 \mathrm{~h}$ at $37^{\circ} \mathrm{C}$.

Once the samples were dried, they were weighed one by one, recording its weight as $W_{f}$.

The following equation was used for the weight measurements and expressed in \% Dry weight remaining:

$$
\% \text { Dry weight remaining }=\left(\frac{W_{f}}{W_{i}}\right) \times 100
$$

For comparison purposes, scaffolds of just chitosan medium molecular weight (MMW) were prepared via freeze- gelation methodology.

\section{Cytotoxicity}

Cytotoxicity was evaluated through Alamar Blue assay. This method tests cell health and viability under the premise that living cells reduce the non-toxic reagent resazurin (blue and non-fluorescent) to resorufin (red and fluorescent). Therefore, an estimation of the living cells on time is performed by measuring the absorbance of their media.

After disinfection of the scaffolds with $70 \%$ ethanol and $1 \mathrm{~h}$ under UV light, 30,000 cells of the U2OS cell line were seeded onto the scaffolds in a 24-well plate. On 1st, 4th, 9th and 14th days Alamar blue assay was performed by changing the media, where the scaffolds were suspended and the reagent (10\% of the sample volume) added. By using a plate reader, the absorbance of each sample was obtained (reading at $570 \mathrm{~nm}$ ). Viability was estimated using the percentage of metabolic activity of the cells. Considering as $100 \%$ of metabolic activity the resultant absorbance for the cells growing in the well-plate without heparinized scaffold, and $0 \%$ the result for the media alone. Media was changed once a week and after every evaluation.

\section{Angiogenic activity ex-ovo CAM Assay}

During ex-ovo CAM assay (CAM stands for chorioallantoic membrane) the scaffolds are implanted on live chicken embryo. This assay provides an overview of the performance of the material in a live environment. The procedure used in this study was based on Eke G. et al. (2017) [35]. Fertilized chicken eggs were carefully cleaned with 20\% IMS solution prior to its incubation at $37.5^{\circ} \mathrm{C}$ in a humidified chicken incubator. On the third day of incubation, the eggs were carefully cracked, the embryo was transferred into a sterilized petri dish and kept in a humidified incubator at $37.5^{\circ} \mathrm{C}$. The survival of the embryos as well as the consistency of the parameters (temperature, humidity) were checked daily during the experiment.

The scaffolds were sterilized using $70 \%$ ethanol and 1 $\mathrm{h}$ under UV light, prior to their implantation on the CAM of the embryo on the 7th day. Sample pictures were obtained on the 10th day and on the sacrifice day (13th day), using an optical microscope.

\section{CAM histology}

The scaffolds were retrieved from the chicken embryo on the sacrifice day by cutting the surrounding CAM tissue with the scaffold. These materials were fixed using formaldehyde solution $3.7 \%$ for $12 \mathrm{~h}$. The fixed scaffold was sliced using cryo-embedding technique (Leica). Conventional haematoxylin and eosin $(\mathrm{H} \& \mathrm{E})$ staining was performed. The slices were then examined under light microscope to observed blood vessel infiltration in the CAM.

\section{Results and discussion Scaffolds}

The appearance of the freeze-gelled scaffolds was of porous white sponges (Fig. 1).

Scaffolds of disc shape of $5 \mathrm{~mm}$ diameter and $2.5 \mathrm{~mm}$ thickness approximately were produced. These scaffolds exhibited an average weight of $6.47 \pm 0.076 \mathrm{mg}$ ( $\mathrm{SD}=$ 1.014). Concentrations of heparin loading is given in Table 1.

\section{SEM characterization}

Morphology of the freeze-gelled scaffolds was observed by SEM. The physical structure confirmed interconnected porosity and the presence of embedded hydroxyapatite crystals distributed throughout the polymer matrix were also visible. As observed in Fig. 2, neither the process of heparin loading by immersion in aqueous solution nor the concentration of heparin loaded affected the structure of the scaffold, as no visible changes were observed among scaffolds with different concentrations.

The notable differences were spotted on the porosity and distribution according to the side of the disc-shaped scaffold. Figure 3 presents images from the scaffold loaded with $0.5 \mathrm{mg} / \mathrm{mL}$ for illustration purposes, all the scaffolds exhibited a similar behaviour. Chitosan-rich side was found on the top side of the scaffold and was characterised for a porosity ranging from 90 to $195 \mu \mathrm{m}$, Fig. 3 (A). A smoother surface with scattered hydroxyapatite particles could be seen. Lateral images of the scaffold, Fig. 3 (B), 


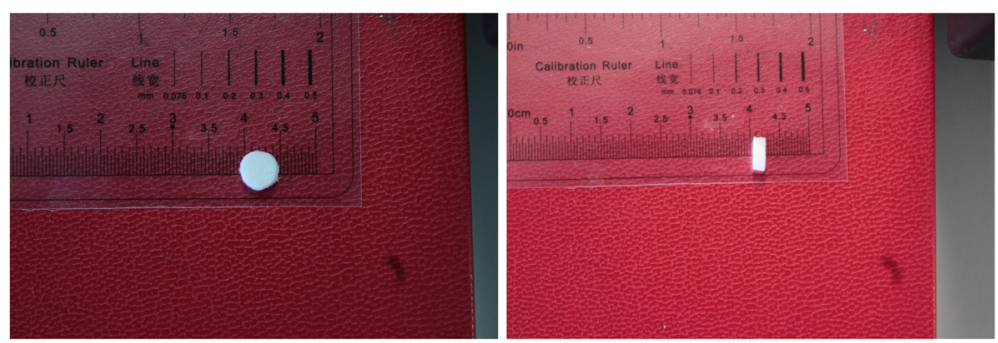

Fig. 1 Freeze-gelled chitosan/ hydroxyapatite scaffolds after heparin loading

show a porosity distribution from 51.61 to $392.63 \mu \mathrm{m}$, with an average of $182.83 \mu \mathrm{m}$. Hydroxyapatite is evenly embedded throughout the chitosan matrix of this side. The bottom face of the scaffold exhibited the hydroxyapatite-rich side, Fig. 3 (C), a more packed appearance can be observed, with an average pore size of $40 \mu \mathrm{m}$. The entire surface of the polymer matrix appears to be covered by the hydroxyapatite particles.

This gradient of hydroxyapatite from top to bottom originated during freezing part of the scaffold formation process. Hydroxyapatite surface charge usually is slightly negative, due the presence of hydroxy and phosphate groups on its lattice surface. However, this charge can change with $\mathrm{pH}$ [36]. When hydroxyapatite becomes in contact with an acidic solution, there is a change on its surface charge due to the adsorption of $\mathrm{H}^{+}$onto the hydroxyl ions and the protonation of the phosphate groups of the surface [37]. Despite this change on its surface, it has affinity towards chitosan due to its positive charge, which also prevents the precipitation and keeps molecules dispersed within the chitosan matrix. Additionally, hydroxyapatite is not soluble in the solution in which chitosan was dissolved, therefore, this chitosan solution is used to form a matrix in which the phosphate ions may be suspended. During the process of freeze-gelation pores were formed by removing the solvent while the frozen shape was retained. The freezing part of the process was kept at a relatively slower rate to, giving sufficient time for the crystals to form and generate pores

Table 1 Materials with variable heparin concentrations

\begin{tabular}{lll}
\hline Sample Name & Matrix Composition & $\begin{array}{l}\text { Heparin loading } \\
\text { concentration } \\
(\mathbf{m g} / \mathbf{m L})\end{array}$ \\
\hline MMW & Chitosan MMW & - \\
M0 & Chitosan MMW + Hydroxyapatite & - \\
M0.5 & Chitosan MMW + Hydroxyapatite & 0.5 \\
M1 & Chitosan MMW + Hydroxyapatite & 1 \\
M2 & Chitosan MMW + Hydroxyapatite & 2 \\
M5 & Chitosan MMW + Hydroxyapatite & 5 \\
\hline
\end{tabular}

within the chitosan matrix. Therefore, during this process, an amount of the suspended hydroxyapatite settled at the bottom of the container, while the rest was kept in suspension and could be observed homogeneously dispersed in rest of the scaffold.

Hydroxyapatite rich side of the scaffold allows to exploit its' bioactive property. Since hydroxyapatite is often used as coating to induce bioactivity on orthopaedic implants, mainly because of the interaction of the osteoblasts around the bioactive surface, which promotes the formation of new mineralised bone matrix.

Pore size represents a very important parameter for designing any scaffold in tissue engineering. It influences the cellular activity in terms of attachment, matrix deposition and differentiation. It seems to exist a general agreement within the field of bone tissue engineering to consider that the optimal pore size for bone tissue scaffolds is between 100 and $500 \mu \mathrm{m}$, to have good cell adhesion and proliferation, and vascular ingrowth $[38,39]$.

\section{FTIR characterization}

Chemical composition was characterised by ATR-FTIR spectroscopy. Top and bottom surfaces of the disc-shaped scaffolds were analysed, showing the formation of a functionalized scaffold, with a chitosan-rich surface and a hydroxyapatite-rich surface, as described in the previous section. Figure 4 shows the resulting spectra comparing both surfaces of the scaffold and the precursors used (chitosan and hydroxyapatite). FTIR spectra confirmed the composition of the scaffold and, in accordance with the SEM results, show the clear difference between the two surfaces. For comparison purposes hydroxyapatite spectrum (C) was placed right below the bottom surface spectrum (D), the same case with chitosan (A) and the top surface (B).

Hydroxyapatite and the bottom surface showed welldefined phosphate $\left(\mathrm{PO}_{4}\right)$ peaks at $564 \mathrm{~cm}^{-1}$ and 1018 $\mathrm{cm}^{-1}$, corresponding to phosphate $v_{4}$ and $v_{3}$ stretching modes, respectively. Likewise, hydroxyl $(\mathrm{OH})$ stretch peak was observed at $3571 \mathrm{~cm}^{-1}$ for HA spectra [40].

Chitosan was present on the top surface and the corresponding spectra showed a band for alkyl $\left(\mathrm{CH}_{2}, \mathrm{CH}_{3}\right)$ 


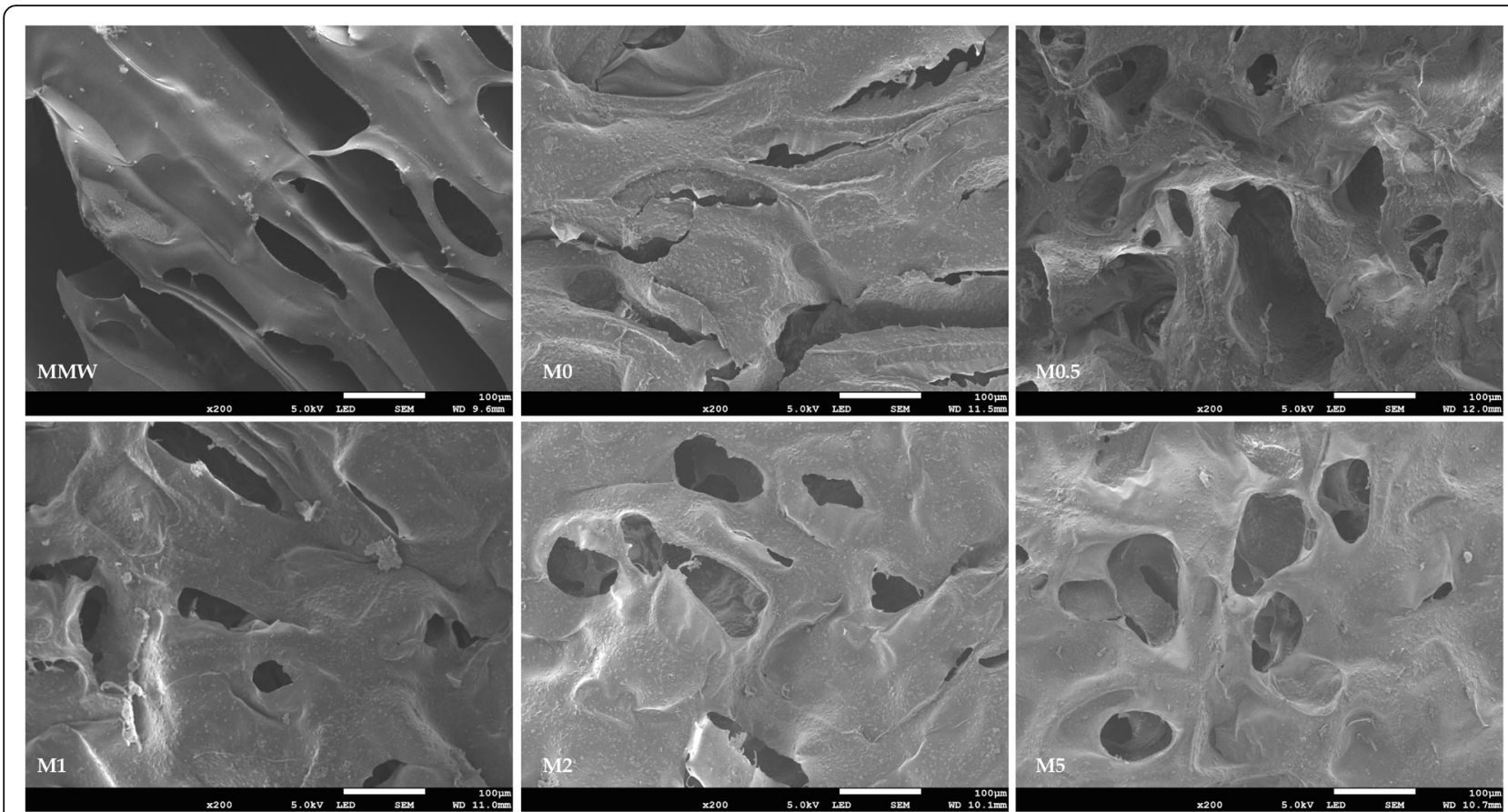

Fig. 2 SEM images showing that the porous structure of the obtained scaffolds is similar regardless of the heparin content. We present chitosan surface. Magnification 200X, all scales bars are $100 \mu \mathrm{m}$

bending at $1375-1415 \mathrm{~cm}^{-1}$, a small hump between 1586 and $1650 \mathrm{~cm}^{-1}$ corresponding to the $\mathrm{CO}$ stretching from Amide I, and, the peaks of $\mathrm{CH}_{2}, \mathrm{CH}_{3}$ stretch near $2850-2915 \mathrm{~cm}^{-1}[24,41]$. These spectra also showed a band between 1025 and $1060 \mathrm{~cm}^{-1}$ assigned to C-O-C stretching and a broad peak at $3555 \mathrm{~cm}^{-1}$, which may be attributed to a combination of $\mathrm{OH}$ and $\mathrm{NH}$, stretching vibrations $[24,41]$.

\section{Heparin loading determination}

Toluidine Blue assay allowed the quantitative determination of the heparin content as well as its qualitative distribution on the scaffold. The distribution throughout the scaffold was uniform, showing the change of colour from blue to purple along the chitosan matrix. The change of colour in the Toluidine Blue solution is immediate after the immersion of the scaffolds, with gradual differences in the shades of blue and purple according to the heparin concentration, the colours of the graph intend to mimic the shades observed. From Fig. 5 (A to E), it is possible to notice the difference between the sample without heparin which exhibits a completely blue surface, and the rest of the samples with heparin, which can be seen as purple surfaces with some scattered blue

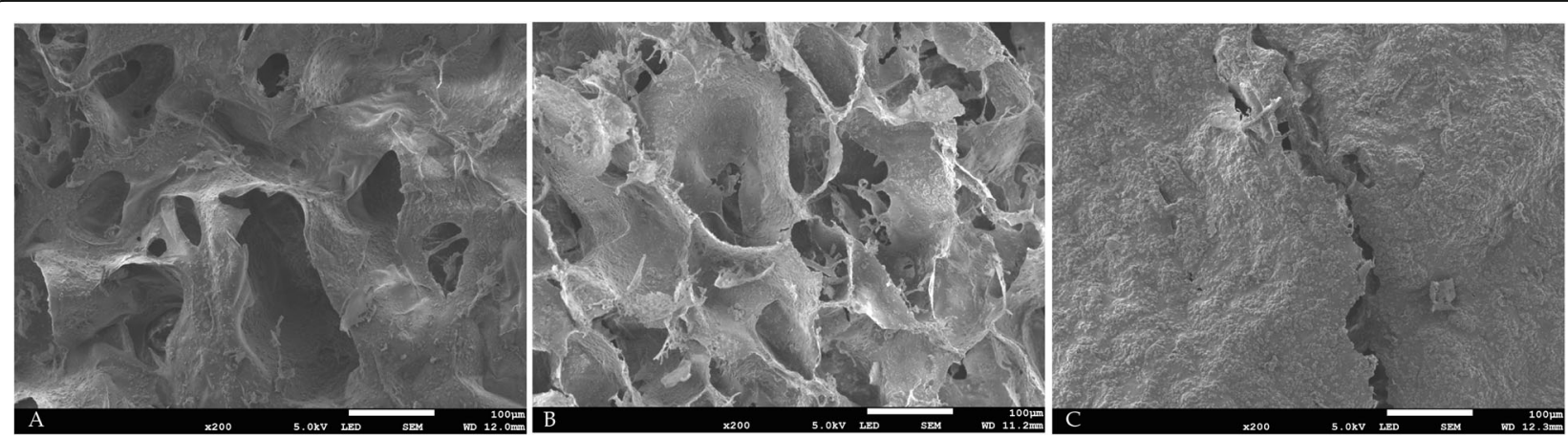

Fig. 3 SEM images showing the porous structure of the obtained scaffolds. It is possible to observe the gradient on the content of hydroxyapatite from A (Top surface) to C (Bottom surface), B is the image from the lateral side of the scaffold. Images of the scaffold loaded with $0.5 \mathrm{mg} / \mathrm{mL}$ are presented as an example of hydroxyapatite gradient in the scaffolds, similar results were observed in all the scaffold containing hydroxyapatite. Magnification 200X, all scales bars are $100 \mu \mathrm{m}$ 


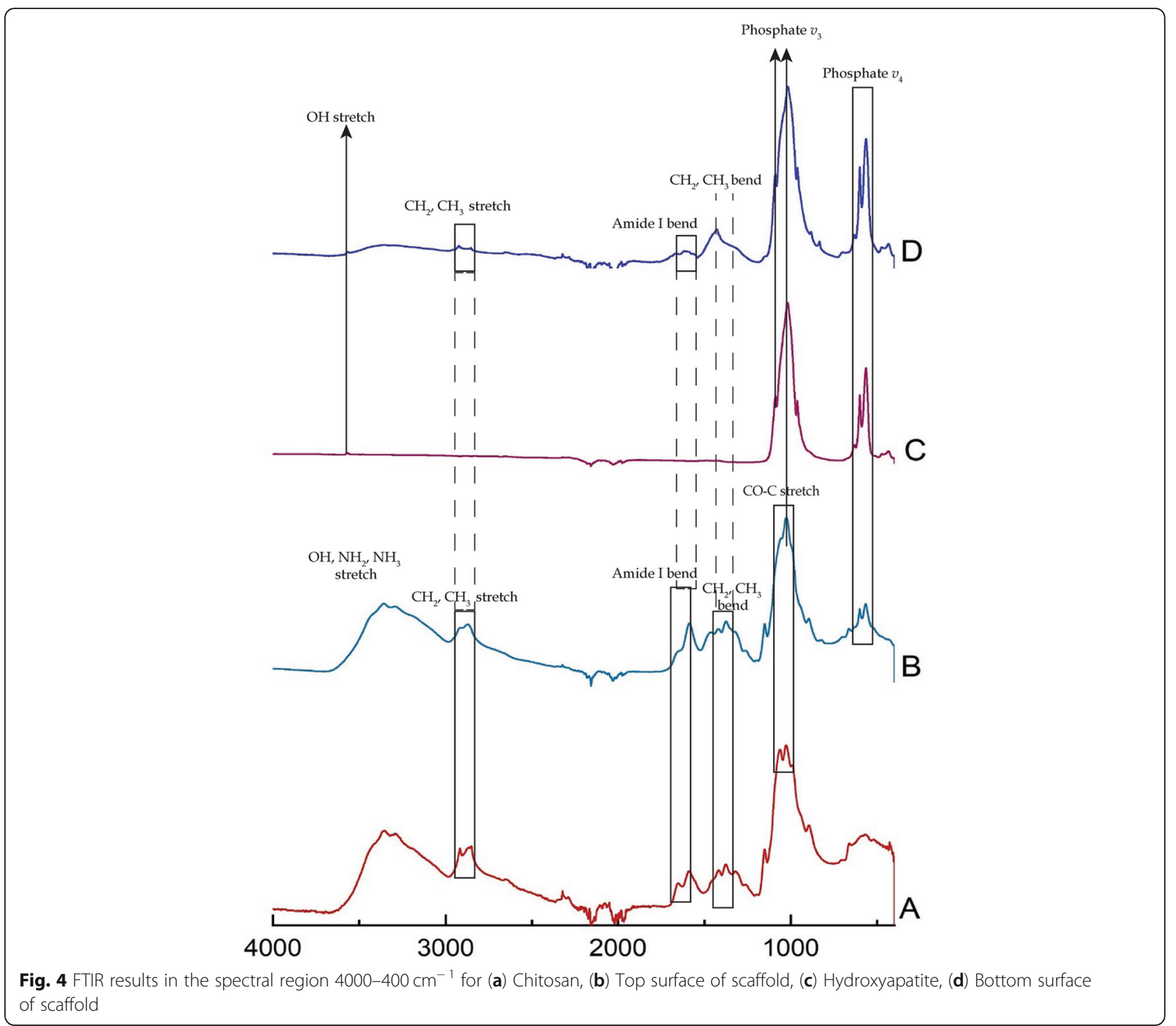

spots. However, the purple shade in the heparinized scaffolds did not give much information about the concentration of each sample, as no apparent difference among scaffolds was notable, regardless of the concentration.

Regarding the quantification of the heparin, Fig. 6 presents a graph showing the amount of heparin released throughout the different intervals. It is possible to observe that the amount of heparin detected on every scaffold varied according to the loading concentration where they were immersed. By using these data, we obtained the total sum of all the results giving us an estimated total concentration of heparin per scaffold, showed in Table 2. Average concentrations from $28.14 \mu \mathrm{g}$ to $315.89 \mu \mathrm{g}$ per scaffold were achieved.
The loading of heparin onto chitosan is feasible due to the presence of positive charge (amine groups) in the polymer matrix and anionic charge (sulphate and carboxyl groups) of heparin. However, it is important to highlight that during this study most of the heparin was released within the first $1.5 \mathrm{~h}$ of immersion in water as shown in Fig. 6. The higher the loading concentration of the scaffold the higher the percentage of heparin content release within the first $1.5 \mathrm{~h}$, with the maximum concentration losing the $90 \%$ of the heparin content within this time. Gümüșderelioğlu \& Aday (2011) reported a fast release with their functionalized chitosan scaffold where heparin had been bonded electrostatically [25]. The functionalization of chitosan scaffolds with heparin was caused by the presence of positive charge (amine groups) in the polymer matrix 


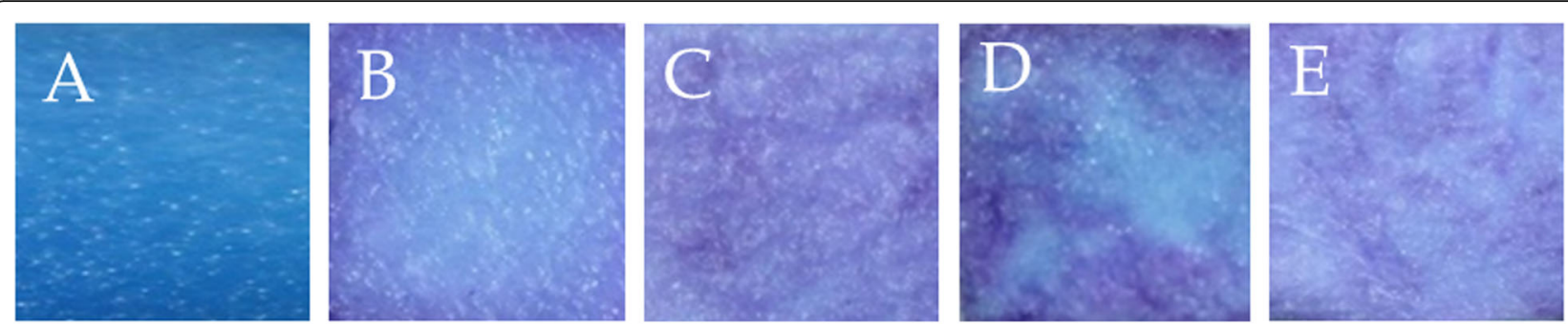

Fig. 5 Scaffolds stained with Toluidine blue; it is possible to observe the change from blue to purple in the presence of heparin. Loading concentrations: (a) No heparin, (b) $0.5 \mathrm{mg} / \mathrm{mL}$, (c) $1 \mathrm{mg} / \mathrm{mL}$, (d) $2 \mathrm{mg} / \mathrm{mL}$, (e) $5 \mathrm{mg} / \mathrm{mL}$

and anionic charge (sulphate and carboxyl groups) of heparin. Therefore, the DD of the chitosan plays an important role on heparin loading, our matrix was prepared with a chitosan with $\mathrm{DD} \geq 90 \%$, thus, presenting enough amino groups for the heparin interaction. The abovementioned authors, however, report a release of at least $50 \%$ within the first 10 days of exposure to cell culture process. This means that the addition of hydroxyapatite, causes the electrostatic interaction between chitosan and heparin to be weaker, since the distribution of hydroxyapatite throughout all chitosan matrix hinders their interaction. Additionally, hydroxyapatite exhibits a slightly negative charge due the presence of phosphate and hydroxyl groups. This negative charge causes a repulsion of between these two components during their interaction in the matrix. This

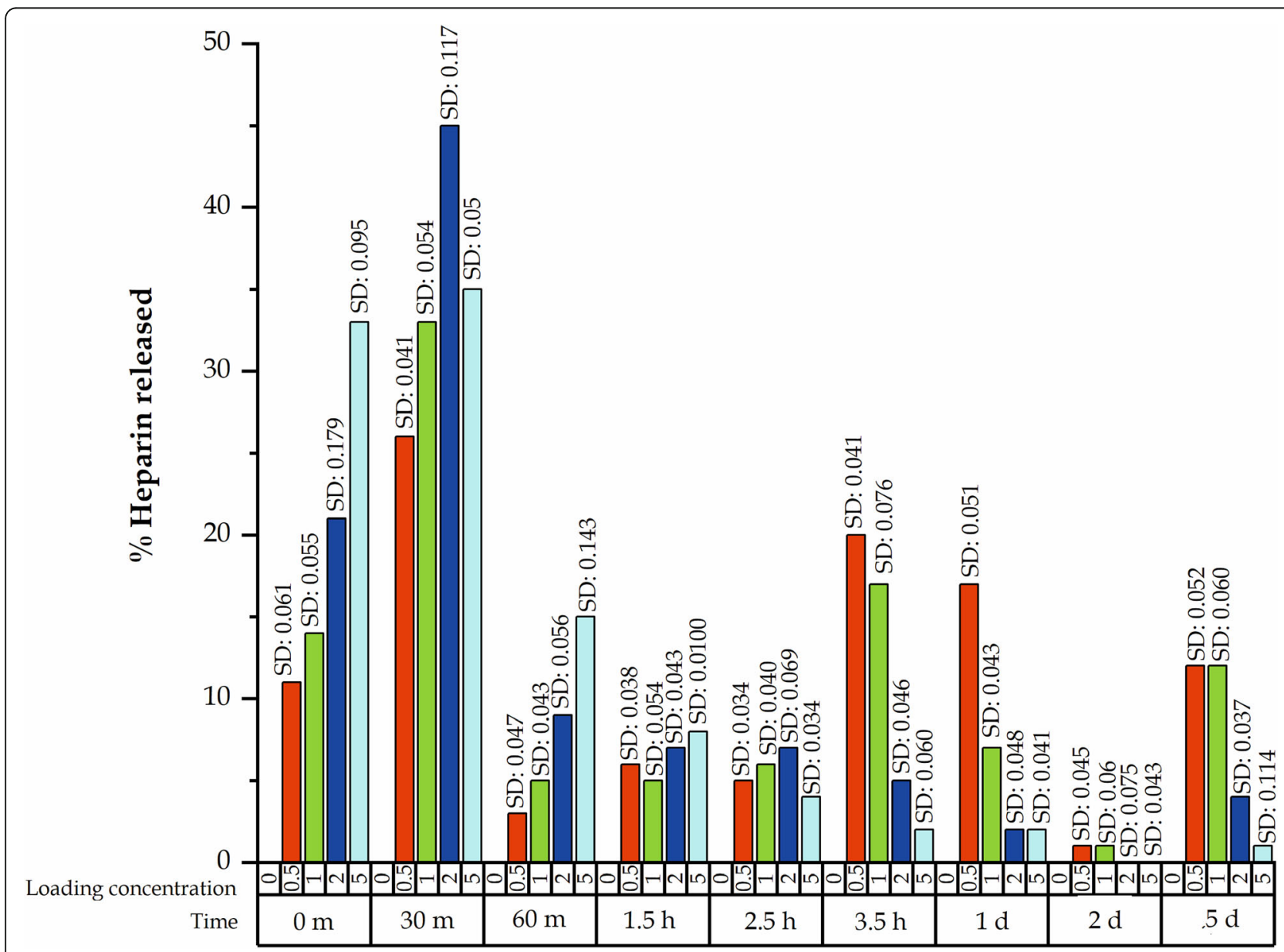

Fig. 6 Heparin release from the scaffolds over time 
Table 2 Average total content per scaffold

\begin{tabular}{lll}
\hline $\begin{array}{l}\text { Loading } \\
\text { Concentration } \\
\mathbf{m g} / \mathbf{~ m L}\end{array}$ & $\begin{array}{l}\text { Approximate Content } \\
\mathbf{\mu g} / \mathbf{s c a f f o l d}\end{array}$ & $\begin{array}{l}\text { \% release within } \\
\text { the first } \mathbf{1 . 5} \mathbf{~ h}\end{array}$ \\
\hline 0.5 & 28.14 & 44.68 \\
1 & 50.14 & 57.06 \\
2 & 138.85 & 82 \\
5 & 315.89 & 90.38 \\
\hline
\end{tabular}

explains the faster release when more heparin was presented. Covalent bonding of heparin would represent an option worth to explore, as well as the response of this bonding and the addition of hydroxyapatite.

It is also important to note that the presence (or release) of heparin from the scaffolds loaded with the lowest loading concentration, was more constant over time. This is notable by day 5 , when they still show the presence of more than $20 \%$ of the total of heparin. Meaning that lower loading concentrations allow a better interaction of the heparin solution with the chitosan/hydroxyapatite matrix and provide a true sustained release for a longer period.

\section{In-vitro degradation}

The purpose of exposing the material to the action of enzyme was to mimic the degradation it undergoes during implantation, lysozyme is present in various human fluids in varied concentrations [42]. For comparison purposes, scaffolds of just chitosan medium molecular weight (MMW) were prepared via freeze- gelation methodology.

Figure 7 shows the variations of the scaffold structure over time under the immersion in the PBS + Lysozyme. It is important to mention that though the presence of heparin and the loading methodology did not affect the scaffold structure as mentioned on section 3.2, when comparing pure chitosan scaffolds with hydroxyapatite scaffolds, the latter present closed porosity, i. e. the porosity looks tighter and the pore size smaller, which means that the presence of hydroxyapatite closes down the pore structure of the chitosan matrix. This is mainly formed when preparing the solution for the scaffolds, as the addition of hydroxyapatite into the chitosan solution, results in a thicker solution due to the attraction between chitosan and hydroxyapatite, as described in section 3.2. This solution results denser zones undergoing freeze-gelation process, and consequently smaller pore

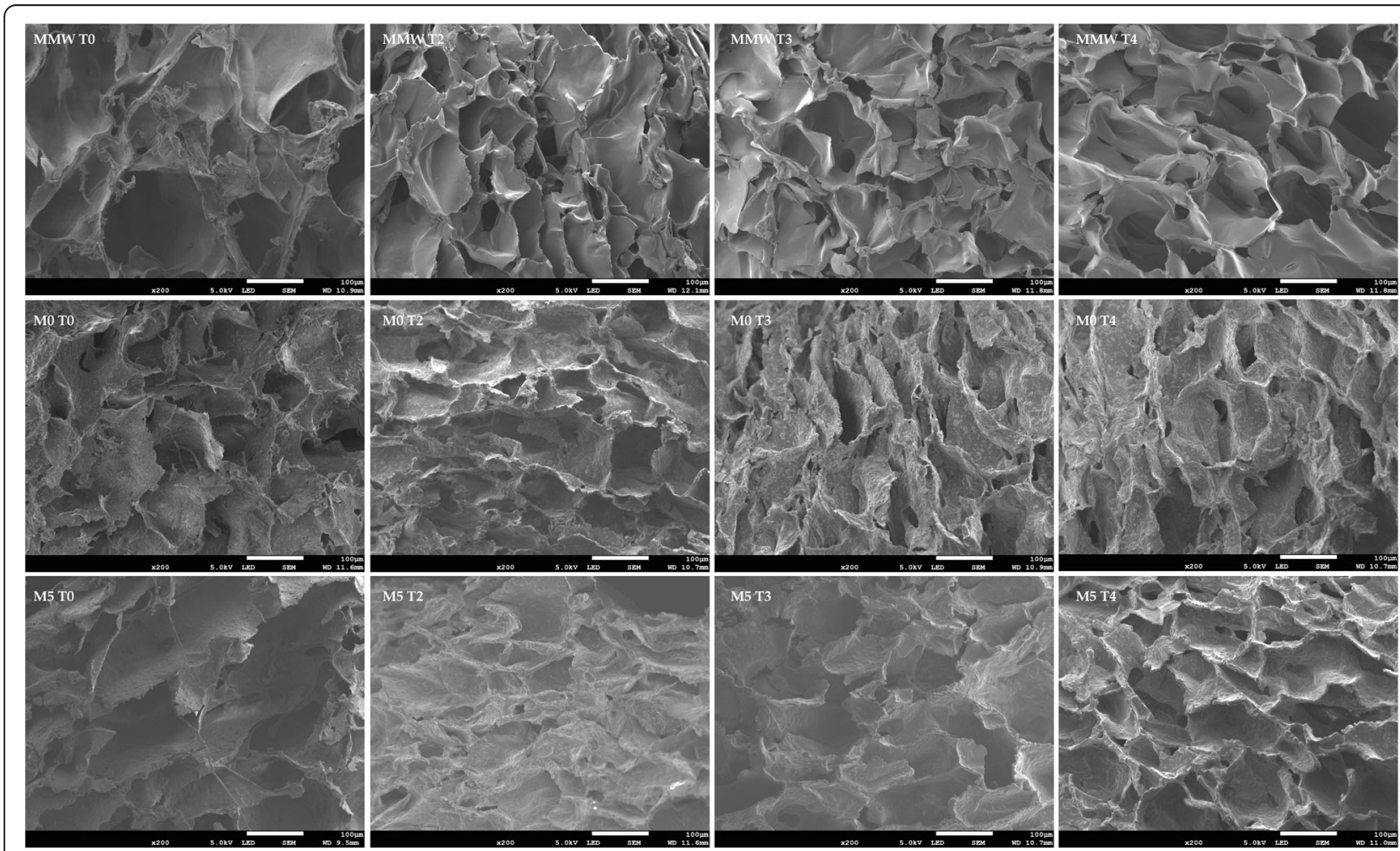

Fig. 7 SEM images of scaffold structure over time immersed in PBS and lysozyme. MMW represents scaffold of only chitosan medium molecular weight, M0 scaffold of chitosan/hydroxyapatite without heparin, and M5 scaffold of chitosan/hydroxyapatite and $5 \mathrm{mg} / \mathrm{mL}$ loading concentration of heparin. T0 before immersion, T2 after 7 days of immersion, T3 after 14 days of immersion, and T4 after 21 days of immersion. All scale bars are $100 \mu \mathrm{m}$ 
sizes. Additionally, during freeze-gelation process, there is some remelting of the solution, caused by the exothermic neutralization with the sodium hydroxide solution for the gelation phase. This slight remelting creates different zones within the frozen solution, some more compact than the others, generating a difference on their porosity [43].

Regarding the influence of degradation media, it was observed that for day 7 there was precipitation of salts all over the scaffolds with hydroxyapatite, particularly for the scaffold with heparin. By day 14 and 21 a similar trend was observed with all the scaffolds, salts precipitation on hydroxyapatite/scaffolds-surface, but all of them presented slightly open porosity, which may be due to the degradation of chitosan matrix. This is evident from the graph presented in Fig. 8, showing the percentage of dry weight remaining after degradation protocol.

Generally, immediately after immersion in liquids, chitosan hydrogels tend to swell and retain water, which can explain little or non-existent weight loss of the first day of immersion. However, with time the swelled structure provides a higher porosity and surface area that favours lysozyme degradation of the structure and loss of the weight and integrity of the scaffold. It was observed in this study only for chitosan scaffold, the weight loss was immediate since lysozyme degrades chitosan by hydrolysing its glucosamine bonds. But in this case, chitosan matrix degradation was affected by the presence of hydroxyapatite, which hampers lysozyme interaction with chitosan. Lysozyme adsorbed onto hydroxyapatite surfaces, favoured by the interactions of lysozyme functional groups and hydroxyapatite phosphate groups [44]. Furthermore, chitosan matrix degradation depends on $\mathrm{pH}$, and the presence of heparin decreases protonation of chitosan amino groups due to its complexation with the negative charged heparin-functional groups, neutralizing $\mathrm{pH}$ with time [45].

Therefore, the behaviour of the scaffolds could be due to the deposition of lysozyme on hydroxyapatite, and the presence of heparin holding up chitosan degradation. In addition, weight loss and a slight yielding of chitosan structure was also observed after 21 days of exposure.

\section{Cytotoxicity}

Cell viability evaluation determines the ability of the material to maintain the cells alive within the scaffold during a certain period. The scaffold should provide a

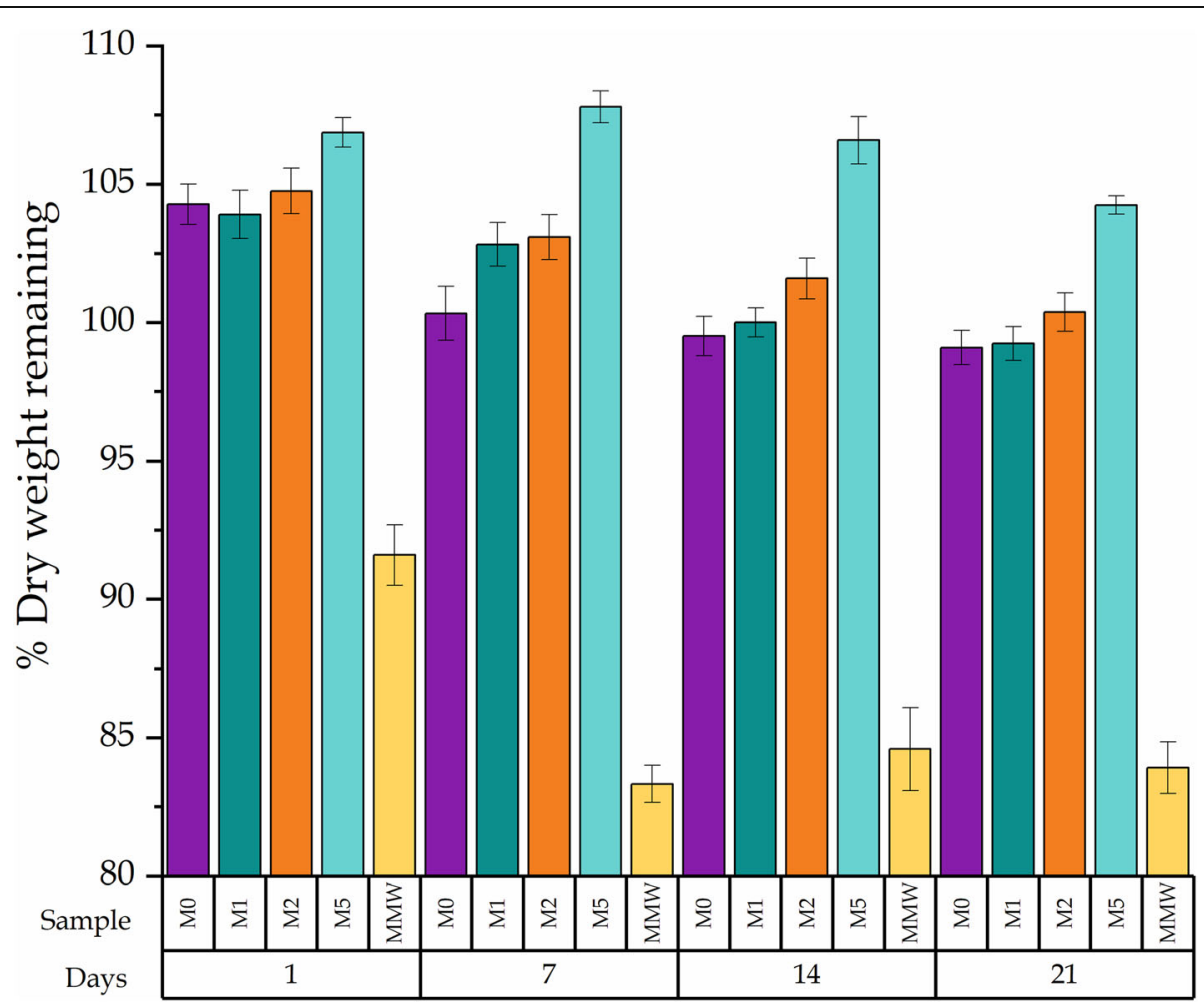

Fig. 8 Dry weight remaining ratio (\%) from scaffolds exposed to PBS and lysozyme over time. $p<0.005$ 


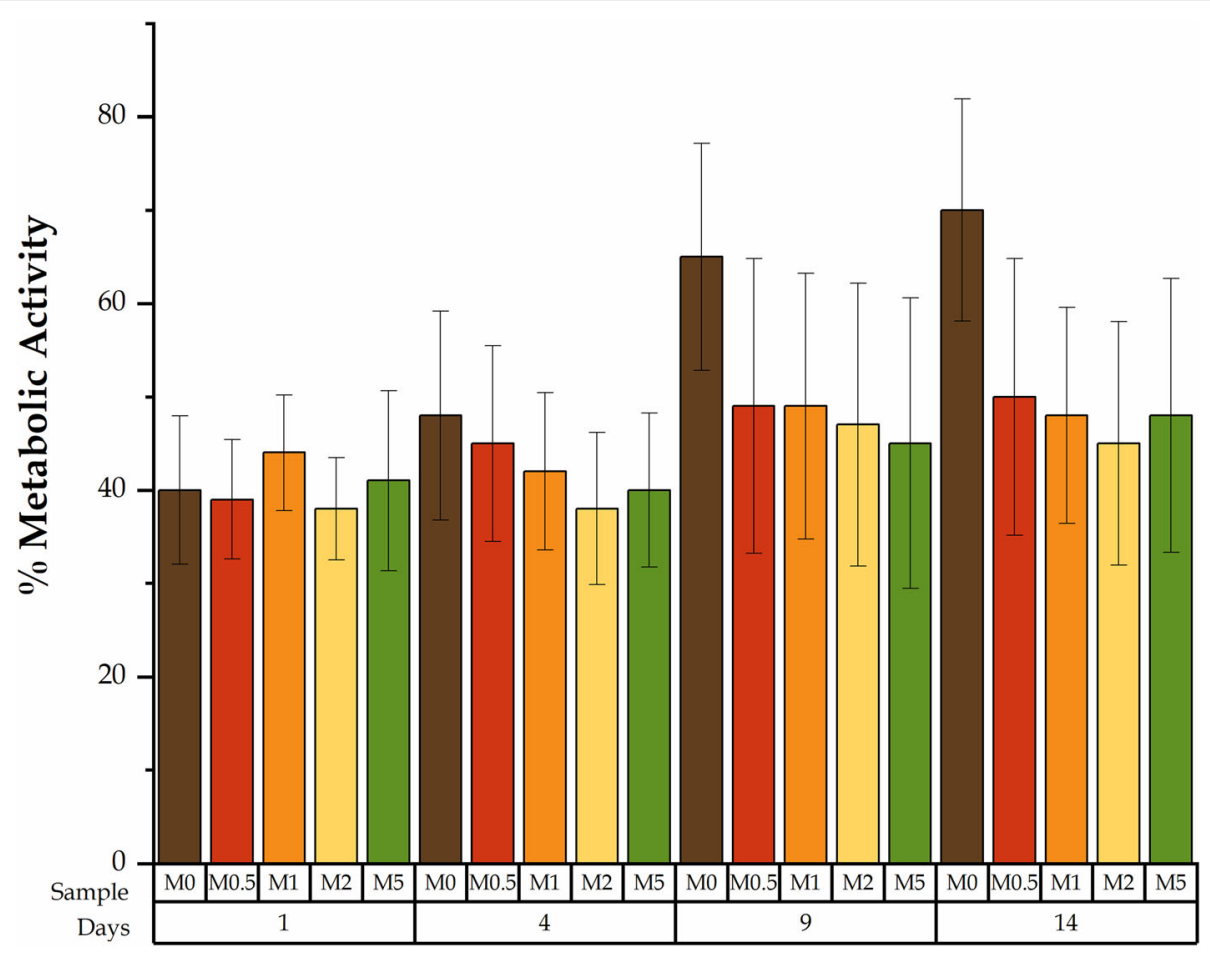

Fig. 9 Metabolic activity of the U2OS cells on the heparinized scaffolds for 14 days. $p<0.005$

suitable environment in terms of attachment and space for their migration. Chitosan and hydroxyapatite scaffolds are already known to offer the appropriate biocompatible characteristics for cell survival [26]. The evaluation of cell viability was performed by means of an Alamar Blue assay. In this method, the measurement of the absorbance in the cell media provides an estimation of living cells, given that living cells reduce resazurin (non-fluorescent, low absorbance) to resorufin (highly fluorescent, high absorbance).

The metabolic activity of U2OS cells on the scaffolds is presented in Fig. 9. The cells were evaluated for 14 days, with readings taken on the 1st, 4th, 9th and 14th day. According to these results, regardless of the heparin content, cell activity remains constant throughout the entire evaluation. Proving cell attachment, during the first day, and cell survival, until the last day of the assay. It can be observed that for the scaffolds without heparin, the proliferation of cells was greater in time (higher metabolic activity) when compared to the behaviour observed in the scaffolds with different heparin concentrations.

Furthermore, the heparin concentration hinders cells proliferation. Therefore, we observe that higher heparin contents result in less metabolic activity. Our scaffolds present a fast desorption of heparin, as mentioned in the previous section, which does not allow heparin to interact with fibronectin and other attachment factors [25], causing the low proliferation profile in these results. Similar results were presented by Gümüșderelioğlu \& Aday (2011), scaffolds with electrostatically loaded heparin reporting no particular proliferation during their study, however, showing important cell differentiation results [25].

\section{Angiogenic activity ex-ovo CAM Assay}

The CAM functions as a gas exchanger, and a disposer for the chick embryo's waste. It also plays part in the mineral transport for the bone development of the embryo. These activities are mainly due to its location and its highly vascularized nature [46]. Ribatti (2017) describes CAM assay as an outstanding model

Table 3 Vascular index results according to loading concentration

\begin{tabular}{ll}
\hline Sample & Vlx \\
\hline M0 & 33.5 \\
M0.5 & 36.8 \\
M1 & 32 \\
M2 & 33 \\
M5 & 28.5 \\
\hline
\end{tabular}




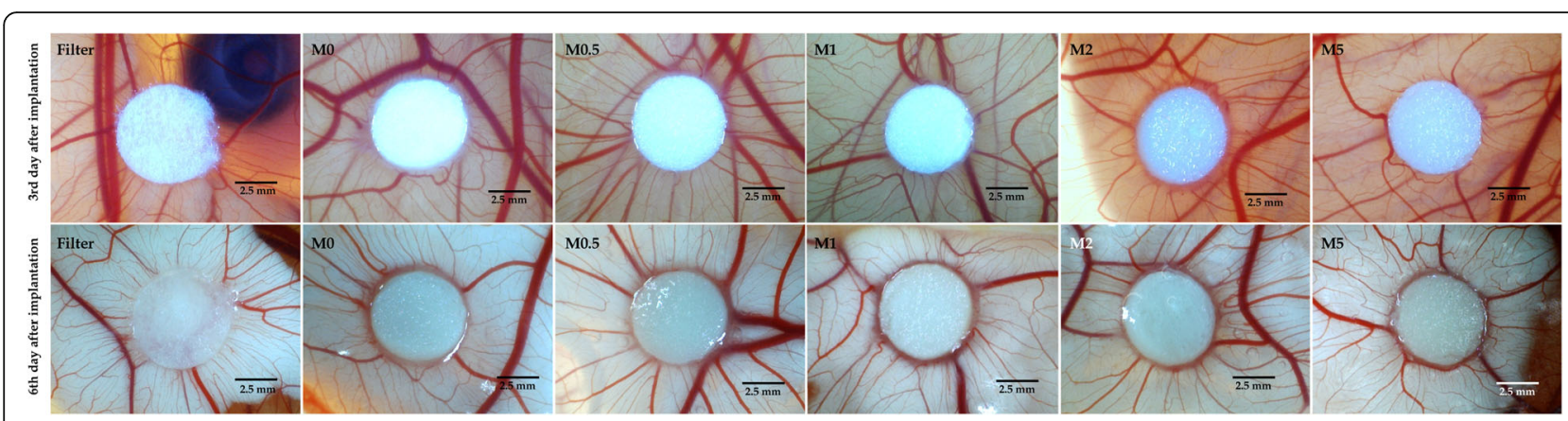

Fig. 10 Micrographs of the samples of the different concentrations implanted on the CAM at the 3rd and the 6th day of implantation

to assess angiogenic performance of materials and drugs [47], and Mangir et al. (2019) and Eke et al. (2017) highlighted the use of the ex-ovo technique to improve the visual analysis, monitoring, and comparison of the materials under evaluation [35, 48].

CAM assay allowed us to obtain preliminary performance of the heparin-loaded scaffolds. Chick embryo health and survival, as well as changes in the vascularization surrounding or attaching the scaffolds, provide useful information on the angiogenic activity of the scaffolds produced in this study.

Micrographs of the scaffolds were taken on the third and the sixth day after the implantation. These images were used to count the blood vessel growing towards the scaffolds, using the method described by Barnhill et al. (1983) for the calculation of the vasculogenic index (VIx). The vasculogenic index represents the number of blood vessels attached to the scaffold creating a steering wheel pattern [49]. Table 3 shows a comparison of the average vasculogenic indexes for the different loading concentrations of the heparinized scaffold.

From the vasculogenic index it was observed that for most of the concentrations, the results were very similar regardless of the amount of heparin loaded. This indicates, firstly, that our scaffold is able to trigger an angiogenic response, this is mainly due to the presence of hydroxyapatite, since its bioactive properties provide the ability to interact with several molecules from the microvascular cells and their surface, improving and promoting their migration and attachment.

On the other hand, a slightly better performance from the scaffold loaded in the $0.5 \mathrm{mg} / \mathrm{mL}$ solution indicating that this loading concentration provided better angiogenic response. Similar results were observed from parallel project within our research group with injectable heparinized-chitosan hydrogels.

However, visually this is not completely notable, as we observed in Fig. 10.
It is important to mention that for the highest heparin loading concentration, most of the embryos died, (survival rate of 20\%). Many of them showed bleeding (leaky) vessels surrounding the scaffold as showed in Fig. 11. Bleeding can be consireded an indicator of heparin overdose [50].

Whereas, the heparin release results for lower loading concentration provided a constant and sustained heparin release, providing heparin availability longer than the rest of the concentrations and without 'overdosing' the implanted area.

During angiogenesis, endothelial tip cells follow the angiogenic stimulus (mostly VEGF), while the proliferating endothelial cell follow the lead of the tip cells elongating the capillary sprout. With time, the new tubular structures will fuse to allow blood flow. According to the study by Ito \& Claesson-Welsh (1999) [21], heparin contributes to present VEGF and FGF to their receptors in the cells, therefore a sustained availability provides enough time for heparin to bind with surrounding angiogenic factors, creating a

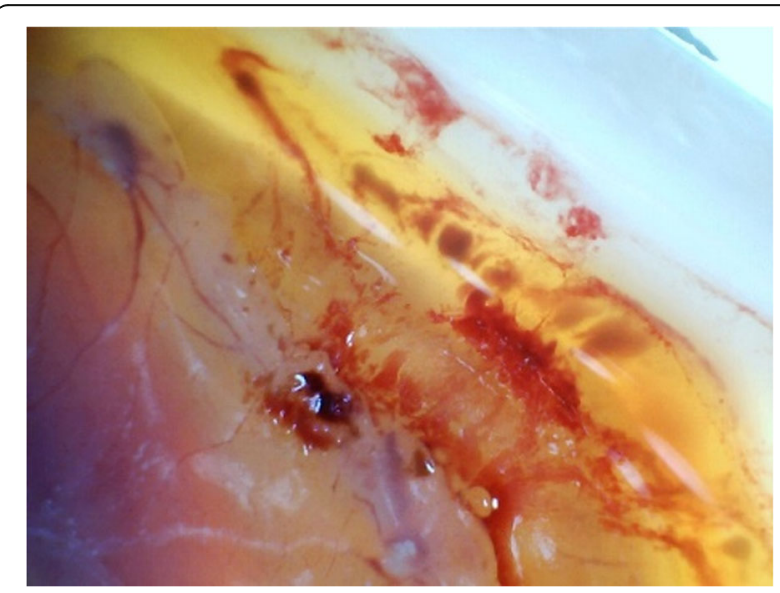

Fig. 11 Spontaneous bleeding of an embryo implanted with the scaffolds with the highest concentration 


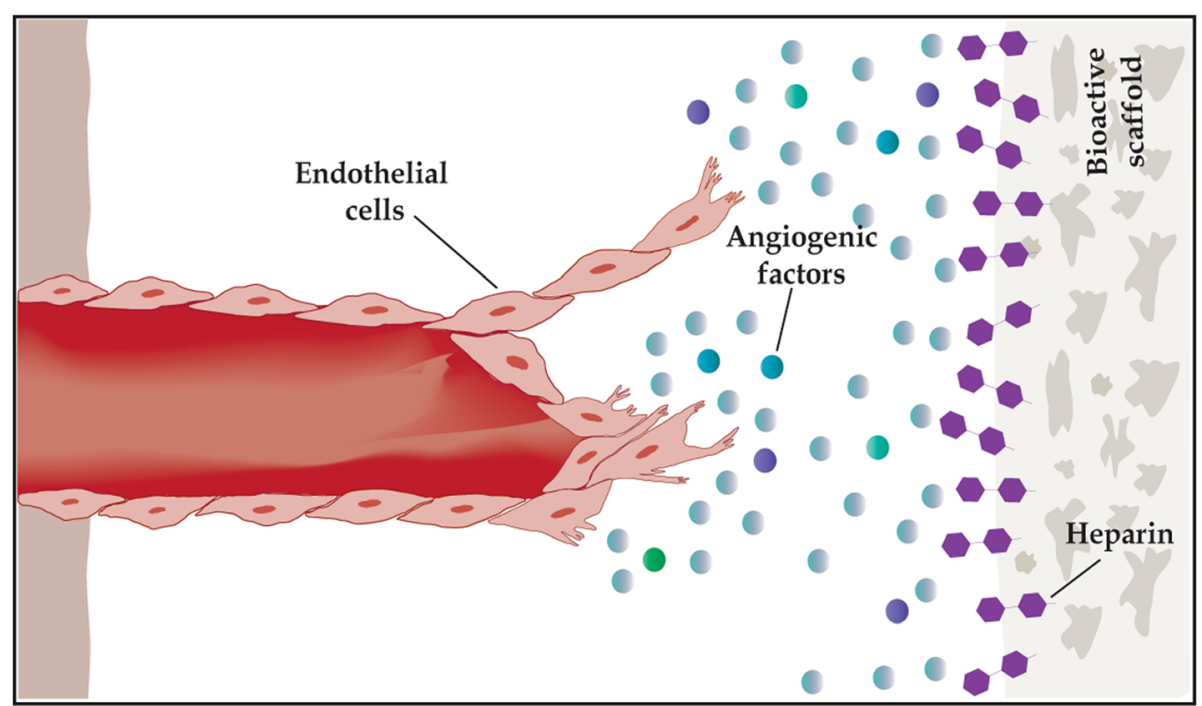

Fig. 12 The heparinized bioactive scaffolds will provide a sustained availability of angiogenic factors, promoting the process of angiogenesis

gradient that will stimulate an angiogenic response (Fig. 12).

\section{CAM histology}

The image in Fig. 13 shows the result of the H\&E staining for the retrieved samples of the CAM assay. In general, integration of the CAM tissue and the scaffold and the appearance of secondary vessels surrounding the CAM tissue (yellow arrows) was observed. No anomalies or appearance of inflammatory cells was detectable.

It is important to observe cellular infiltration into the scaffolds. It may also be stated that for the scaffolds with higher loading concentrations, the integration is not well defined, and the presence of blood vessels appear to be messy.

\section{Conclusions}

In this study, freeze-gelated chitosan/hydroxyapatite scaffolds were prepared. The resultant materials exhibited interconnected porosity, with pore size able to support vascularization ingrowth. Heparin was electrostatically loaded into the porous scaffolds to obtain bioactive bone tissue engineering scaffolds that can significantly promote angiogenesis. A weak interaction was observed between chitosan and heparin, due to the addition of hydroxyapatite in our scaffolds. However, it was observed that heparin was able to delay chitosan degradation in biodegradation analysis, confirming that surface charge plays a pivotal role in final properties of resultant bioactive scaffolds.

The resultant porous scaffolds did not exhibit cytotoxic effects. The results of the CAM assay confirmed the stimulation of angiogenesis and vascular attachment to the synthesized scaffolds. Low heparin concentrations demonstrated a sustained heparin release, exhibiting the best angiogenic responses with concentration of $28 \mu \mathrm{g}$ per scaffold. These materials hold promising potential to be used as pro-angiogenic bone regeneration materials in clinic.

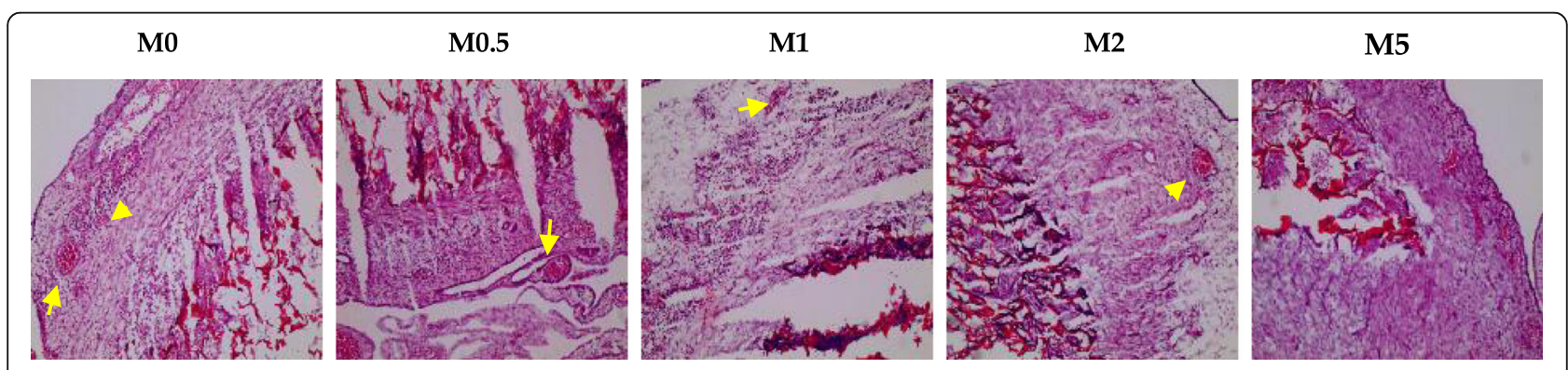

Fig. 13 H\&E staining of the samples for the different sample concentrations. Arrows point to the appearance of secondary blood vessels 


\section{Abbreviations}

DD: Degree of Deacetylation; IU: International Units; CAM: Chorioallantoic Membrane; VEGF: Vascular Endothelial Growth Factor; FGF: Fibroblast Growth Factor; AGF: Angiogenic Roth Factor; LMWH: Low Molecular Weight Heparin; HMWH: High Molecular Weight Heparin; SEM: Scanning electron Microscopy; FTIR: Fourier Transform Infrared spectroscopy; SD: Standard Deviation

\section{Acknowledgements}

We thank Mexican National Council for Science and Technology (CONACYT) scholarship number CVU-410600 for the funds provided.

Authors would like to thank Sara Baldoc from Lancaster University for her kind help with the SEM imaging.

\section{Authors' contributions}

Griselda V Nájera-Romero PhD researcher, who carried out the work and drafted this manuscript. Muhammad Yar: Co-supervisor of the PhD study. Ihtesham Ur Rehman: Supervisor of the PhD study. The author(s) read and approved the final manuscript.

\section{Funding}

Mexican National Council for Science and Technology (CONACYT) scholarship number CVU-410600.

\section{Availability of data and materials}

All supporting data is available and provided as figures and tables.

\section{Competing interests}

The authors declare that they have no competing interests.

\section{Author details}

${ }^{1}$ Engineering Department, Faculty of Science and Technology, Lancaster University, Lancaster LA1 4YW, UK. ${ }^{2}$ Interdisciplinary Research Centre in Biomedical Materials, COMSAT, University Islamabad Lahore Campus, Lahore 54000, Pakistan.

\section{Received: 7 August 2020 Accepted: 6 October 2020}

Published online: 31 October 2020

\section{References}

1. E.M. Bueno, J. Glowacki, Biologic Foundations for Skeletal Tissue Engineering, Ericka M. Bueno and Julie Glowacki, Synthesis Lectures on Tissue Engineering, January, 3(1), 1-22 (2011)

2. M. Doblaré, J.M. García, M.J. Gómez, Modelling bone tissue fracture and healing: A review. Eng. Fract. Mech. 71, 1809-1840 (2004)

3. S.G. Pneumaticos, G.K. Triantafyllopoulos, E.K. Basdra, A.G. Papavassiliou, Segmental bone defects: From cellular and molecular pathways to the development of novel biological treatments. J. Cell. Mol. Med. 14, $2561-$ 2569 (2010)

4. K.D. Hankenson, M. Dishowitz, C. Gray, M. Schenker, Angiogenesis in bone regeneration. Injury 42, 556-561 (2011)

5. U. Saran, S. Gemini Piperni, S. Chatterjee, Role of angiogenesis in bone repair. Arch. Biochem. Biophys. 561, 109-117 (2014)

6. F. Loi et al., Inflammation, fracture and bone repair. Bone $86,119-130$ (2016)

7. E.A. Bayer, R. Gottardi, M.V. Fedorchak, S.R. Little, The scope and sequence of growth factor delivery for vascularized bone tissue regeneration. J. Control. Release 219, 129-140 (2015)

8. K.W. Lee et al., Sustained release of vascular endothelial growth factor from calcium-induced alginate hydrogels reinforced by heparin and chitosan. Transplant. Proc. 36, 2464-2465 (2004)

9. Q. Wu et al., The effect of heparinized decellularized scaffolds on angiogenic capability. J. Biomed. Mater. Res. - Part A 104, 3021-3030 (2016)

10. S. Teixeira, L. Yang, P.J. Dijkstra, M.P. Ferraz, F.J. Monteiro, Heparinized hydroxyapatite/collagen three-dimensional scaffolds for tissue engineering. J. Mater. Sci. Mater. Med. 21, 2385-2392 (2010)

11. D.B. Pike et al., Heparin-regulated release of growth factors in vitro and angiogenic response in vivo to implanted hyaluronan hydrogels containing VEGF and bFGF. Biomaterials 27, 5242-5251 (2006)

12. U. Freudenberg et al., Heparin desulfation modulates VEGF release and angiogenesis in diabetic wounds. J. Control. Release 220, 79-88 (2015)
13. Y.I. Chung et al., Efficient revascularization by VEGF administration via heparin-functionalized nanoparticle-fibrin complex. J. Control. Release 143, 282-289 (2010)

14. Q. Tan et al., Controlled release of chitosan/heparin nanoparticle-delivered VEGF enhances regeneration of decellularized tissue-engineered scaffolds. Int. J. Nanomedicine 6, 929-942 (2011)

15. D.L. Rabenstein, Heparin and heparan sulfate: Structure and function. Nat. Prod. Rep. 19, 312-331 (2002)

16. R.J. Linhardt, S. Claude, Hudson award address in carbohydrate chemistry. Heparin: Structure and activity. J. Med. Chem. 46, 2551-2564 (2003)

17. P. Chiodelli, A. Bugatti, C. Urbinati, M. Rusnati, Heparin/heparan sulfate proteoglycans glycomic interactome in angiogenesis: Biological implications and therapeutical use. Molecules 20, 6342-6388 (2015)

18. T. Bhakuni et al., Role of heparin and non heparin binding serpins in coagulation and angiogenesis: A complex interplay. Arch. Biochem. Biophys. 604, 128-142 (2016)

19. R.B. Rema, K. Rajendran, M. Ragunathan, Angiogenic efficacy of heparin on chick chorioallantoic membrane. Vasc Cell 4, 8 (2012)

20. A. Collen et al., Unfractionated and low molecular weight heparin affect fibrin structure and angiogenesis in vitro unfractionated and low molecular weight heparin affect fibrin structure and angiogenesis in vitro. Cancer Res. 300, 6196-6200 (2000)

21. N. Ito, L. Claesson-Welsh, Dual effects of heparin on VEGF binding to VEGF receptor-1 and transduction of biological responses. Angiogenesis 3, 159166 (1999)

22. M. Yar et al., Heparin binding chitosan derivatives for production of proangiogenic hydrogels for promoting tissue healing. Mater. Sci. Eng. C 74 347-356 (2017)

23. M. Yar et al., Production of chitosan PVA PCL hydrogels to bind heparin and induce angiogenesis. Int. J. Polym. Mater. Polym. Biomater. 65, 466-476 (2016)

24. L. Shahzadi et al., Triethyl orthoformate covalently cross-linked chitosan(poly vinyl) alcohol based biodegradable scaffolds with heparin-binding ability for promoting neovascularisation. J. Biomater. Appl. 0, 1-12 (2016)

25. M. Gümüdereliolu, S. Aday, Heparin-functionalized chitosan scaffolds for bone tissue engineering. Carbohydr. Res. 346, 606-613 (2011)

26. S.K.L. Levengood et al., Chitosan-based scaffolds for bone tissue engineering. J. Mater. Chem. B 2, 3161 (2014)

27. F. Croisier, C. Jérôme, Chitosan-based biomaterials for tissue engineering. Eur. Polym. J. 49, 780-792 (2013)

28. M. Dash, F. Chiellini, R.M. Ottenbrite, E. Chiellini, Chitosan - a versatile semisynthetic polymer in biomedical applications. Prog. Polym. Sci. 36, 981-1014 (2011)

29. F. Baino, G. Novajra, C. Vitale-Brovarone, Bioceramics and scaffolds: A winning combination for tissue engineering. Front. Bioeng. Biotechnol. 3, 202 (2015)

30. M. Šupová, Substituted hydroxyapatites for biomedical applications: A review. Ceram. Int. 41, 9203-9231 (2015)

31. H.Y. Tai, E. Fu, T.M. Don, Calcium phosphates synthesized by reverse emulsion method for the preparation of chitosan composite membranes. Carbohydr. Polym. 88, 904-911 (2012)

32. S.B. Qasim, R.M. Delaine-Smith, T. Fey, A. Rawlinson, I.U. Rehman, Freeze gelated porous membranes for periodontal tissue regeneration. Acta Biomater. 23, 317-328 (2015)

33. P.K. Smith, A.K. Mallia, Colorimetric method for the assay of heparin immobilized heparin preparations content in. Anal. Biochem. 473, 466-473 (1980)

34. S.B. Qasim et al., In-vitro and in-vivo degradation studies of freeze gelated porous chitosan composite scaffolds for tissue engineering applications. Polym. Degrad. Stab. 136, 31-38 (2017)

35. G. Eke, N. Mangir, N. Hasirci, S. MacNeil, V. Hasirci, Development of a UV crosslinked biodegradable hydrogel containing adipose derived stem cells to promote vascularization for skin wounds and tissue engineering. Biomaterials 129, 188-198 (2017)

36. I.S. Harding, N. Rashid, K.A. Hing, Surface charge and the effect of excess calcium ions on the hydroxyapatite surface. Biomaterials 26, 6818-6826 (2005)

37. S.V. Dorozhkin, Dissolution mechanism of calcium apatites in acids: A review of literature. World J. Methodol. 2, 1 (2012)

38. T. Guda et al., Hydroxyapatite scaffold pore architecture effects in large bone defects in vivo. J. Biomater. Appl. 28, 1016-1027 (2014) 
39. L. Roseti et al., Scaffolds for bone tissue engineering: State of the art and new perspectives. Mater. Sci. Eng. C 78, 1246-1262 (2017)

40. I. Rehman, W. Bonfield, Characterization of hydroxyapatite and carbonated apatite by photo acoustic FTIR spectroscopy. J. Mater. Sci. Mater. Med. 8, 14 (1997)

41. A. Zając, J. Hanuza, M. Wandas, L. Dymińska, Determination of N-acetylation degree in chitosan using Raman spectroscopy. Spectrochim. Acta - Part A Mol. Biomol. Spectrosc 134, 114-120 (2015)

42. A Lončarević, M Ivanković, A Rogina, Lysozyme-induced degradation of chitosan: the characterisation of degraded chitosan scaffolds. J. of Tissue Repair and Regeneration, 1, 12-22 (2017)

43. C.Y. Hsieh et al., Analysis of freeze-gelation and cross-linking processes for preparing porous chitosan scaffolds. Carbohydr. Polym. 67, 124-132 (2007)

44. W.H. Lee, C.Y. Loo, K.L. Van, A.V. Zavgorodniy, R. Rohanizadeh, Modulating protein adsorption onto hydroxyapatite particles using different amino acid treatments. J. R. Soc. Interface 9, 918-927 (2012)

45. A.P. Kusumbe, S.K. Ramasamy, R.H. Adams, Coupling of angiogenesis and osteogenesis by a specific vessel subtype in bone. Nature 507, 323-328 (2014)

46. D. Ribatti, The chick embryo chorioallantoic membrane (CAM). A multifaceted experimental model. Mech. Dev. 141, 70-77 (2016)

47. D. Ribatti, The chick embryo chorioallantoic membrane (CAM) assay. Reprod. Toxicol. 70, 97-101 (2017)

48. N. Mangir, S. Dikici, F. Claeyssens, S. Macneil, Using ex Ovo Chick Chorioallantoic membrane (CAM) assay to evaluate the biocompatibility and Angiogenic response to biomaterials. ACS Biomater Sci. Eng. 5, 31903200 (2019)

49. R.L. Barnhill, T.J. Ryan, Biochemical modulation of angiogenesis in the chorioallantoic membrane of the chick embryo. J. Invest. Dermatol. 81, 485488 (1983)

50. P. Monagle, D.M. Studdert, F. Newall, Infant deaths due to heparin overdose: Time for a concerted action on prevention. J. Paediatr. Child Health 48, 380-381 (2012)

\section{Publisher's Note}

Springer Nature remains neutral with regard to jurisdictional claims in published maps and institutional affiliations.

\section{Submit your manuscript to a SpringerOpen ${ }^{\circ}$ journal and benefit from:}

- Convenient online submission

- Rigorous peer review

- Open access: articles freely available online

- High visibility within the field

Retaining the copyright to your article

Submit your next manuscript at $\boldsymbol{\nabla}$ springeropen.com 